\title{
A PARTIAL ORDERING ON SLICES OF PLANAR LAGRANGIANS
}

\author{
PHIL EISEMAN, JONATHAN D. LIMA, JOSHUA M. SABLOFF, \\ AND LISA TRAYNOR
}

To V.I. Arnold, with respect and appreciation.

\begin{abstract}
A collection of simple closed curves in $\mathbb{R}^{3}$ is called a negative slice if it is the intersection of a flat-at-infinity planar Lagrangian surface and $\left\{y_{2}=a\right\}$ for some $a<0$. Examples and non-examples of negative slices are given. Embedded Lagrange cobordisms define a relation on slices and in some (and perhaps all) cases this relation defines a partial order. The set of slices is a commutative monoid and the additive structure has an interesting relationship with the ordering relation.
\end{abstract}

\section{INTRODUCTION}

As part of his study of geometric optics, Arnold introduced the notion of an immersed Lagrange cobordism in $T^{*}(B \times[0,1])$ between immersed Lagrangians in the projection of the boundary to $T^{*}(B \times\{0\})$ and $T^{*}(B \times\{1\})$, [1]. The equivalence classes of oriented immersed Lagrangians up to immersed Lagrange cobordism form a group, which Arnold computed in the case of Lagrangian curves in $\mathbb{R}^{2}$ to be $\mathbb{Z} \oplus \mathbb{R}$, where the $\mathbb{Z}$ records the Maslov class and the $\mathbb{R}$ records the signed area bounded by the curve. Note that these invariants are both homological in nature; in general, immersed Lagrange cobordism obeys an $h$-principle [4], and hence computations of the groups can be approached using algebraic topology (see [2]). That is, immersed Lagrange cobordism is a "flexible" phenomenon in symplectic topology. Passing to embedded Lagrange cobordism between embedded Lagrangians, in contrast, yields a "rigid" theory: embedded Lagrangians in $\mathbb{R}^{2}$ are simply circles, and Chekanov proved that all cobordisms must be cylinders between circles of equal area [3]. In particular, the cobordism group is the free abelian group generated by $\mathbb{R}_{>0}$. Further rigidity is evidenced by Eliashberg's result that there is a unique Lagrange cobordism (up to isotopy) between two circles of the same area [5]. In this paper, we will consider an intermediate situation: we insist that the cobordisms be embedded, but the Lagrangians at the ends may be immersed (in fact, we will record the three-dimensional configuration of the boundary). As we shall see, cobordisms give rise to a relation between their ends that is no longer

$\mathrm{PE}$ and JDL were supported as undergraduate summer research students by the Haverford College faculty support fund. 
an equivalence relation, but rather a partial order in certain (and perhaps all) cases.

More precisely, the objects we study are "slices" of "flat-at-infinity" Lagrangian submanifolds of $\mathbb{R}^{4}$. Consider $\mathbb{R}^{4}$ with the standard symplectic form $d x_{1} \wedge d y_{1}+d x_{2} \wedge d y_{2}$. Let $L_{0}$ denote the Lagrangian $x_{1} x_{2}$-plane in $\mathbb{R}^{4}$, which can also be thought of as the zero-section of $T^{*} \mathbb{R}^{2}$. We say that an embedded Lagrangian submanifold is planar if it is diffeomorphic to $\mathbb{R}^{2}$; a planar Lagrangian is flat-at-infinity if it agrees with $L_{0}$ outside a compact subset of $\mathbb{R}^{4}$. For example, the graph of the differential of any compactly supported smooth function $F: \mathbb{R}^{2} \rightarrow \mathbb{R}$ is a flat-at-infinity planar Lagrangian. It will be convenient to denote by $L_{a}$ the intersection of a Lagrangian $L$ with the hyperplane $\left\{y_{2}=a\right\}$. Let $i_{a}: \mathbb{R}^{3} \rightarrow\left\{y_{2}=a\right\} \subset \mathbb{R}^{4}$ denote the inclusion. Then a link $S \subset \mathbb{R}^{3}$ is a (generic) negative Lagrangian slice if there exists a flat-at-infinity planar Lagrangian $L \subset \mathbb{R}^{4}$ and an $a<0$ such that $L$ is transverse to the hyperplane $\left\{y_{2}=a\right\}$ and $L_{a}=i_{a}(S)$.

Let $\widetilde{\mathcal{S}}$ denote the set of all negative Lagrangian slices together with the empty set. The projection of the non-empty elements of $\widetilde{\mathcal{S}}$ to the $x_{1} y_{1}$-plane will be the unions of immersed curves where each component bounds zero signed area and has winding number 0 . It will be convenient to represent a slice by a "diagram" in the $x_{1} y_{1}$-plane that records the over/under strand with respect to the $x_{2}$-coordinate at double points of the projection. Given a diagram $D$ in the $x_{1} y_{1}$-plane, $\widetilde{D} \subset \mathbb{R}^{3}$ will denote a link that projects to $D$ bijectively at all non-crossing points of $D$; in other words, $\widetilde{D}$ is an $x_{2}$-lift of $D$.

We consider the set of slices up to an equivalence relation: for $S_{1}, S_{2} \in \widetilde{\mathcal{S}}$, say that $S_{1} \sim S_{2}$ if there exists a compactly supported area-preserving diffeomorphism $\varphi$ of the $x_{1} y_{1}$-plane so that $(\varphi \times$ id $)\left(S_{1}\right)=S_{2}$. Let

$$
\mathcal{S}=\widetilde{\mathcal{S}} / \sim
$$

denote the set of equivalence classes of slices. By our equivalence relation, we can work with relatively combinatorial representations of slices, as diagrams need only be defined up to area-preserving diffeomorphism: Figure 1 shows some diagrams of negative slices, while Figure 2 shows some diagrams of closely related unknotted curves that cannot be realized as negative slices; see Sections 3 and 4.3 for proofs.

Embedded Lagrange cobordisms define relations $\triangleleft$ and $\unlhd$ on $\widetilde{\mathcal{S}}$ :

Definition 1.1. Given $S, S^{\prime} \in \widetilde{\mathcal{S}}$, we say that $S \triangleleft S^{\prime}$ if there exists a flat-at-infinity planar Lagrangian $L$ and $a<b<0$ so that $i_{a}(S)=L_{a}$ and $i_{b}\left(S^{\prime}\right)=L_{b}$. The relation $\unlhd$ is defined analogously with $a \leq b<0$.

In Section 2, we will show:

Proposition 1.2. The relations $\triangleleft$ and $\unlhd$ are well-defined on $\mathcal{S}$.

Example 1.3. Using the notation of Figure 1, we have the following relations: 


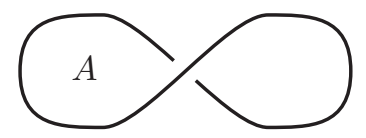

(a)

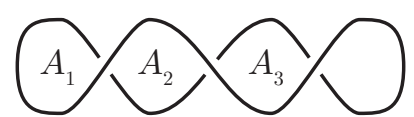

(b)
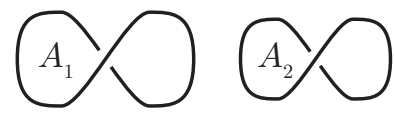

(c)

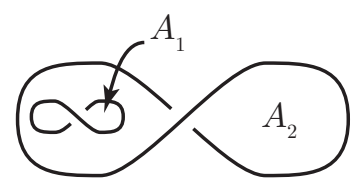

(d)

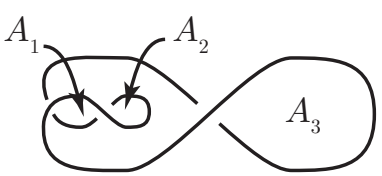

(e)

FiguRE 1 . These diagrams in the $x_{1} y_{1}$-plane represent negative slices of flat-at-infinity planar Lagrangians for some $x_{2^{-}}$ coordinates respecting the crossings. The diagram in (a) will be denoted by $8^{+}(A)$; the diagram in (b) will be denoted by $C^{+-+}\left(A_{1}, A_{2}, A_{3}\right)$; the two component link diagram in (c) will be denoted by $8^{+}\left(A_{1}\right)+8^{+}\left(A_{2}\right)$; the link diagram in (d) will be denoted by $8^{-}\left(A_{1}\right) \odot 8^{+}\left(A_{2}\right)$; and the diagram in (e) will be denoted by $8^{-}\left(A_{1}, A_{2}\right) \times 8^{+}\left(A_{3}\right)$. The positive numbers $A_{i}$ represent the areas of bounded regions of the diagrams.

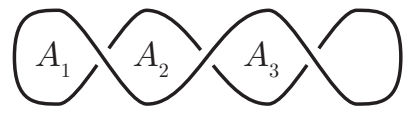

(b)

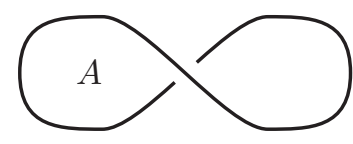

(a)

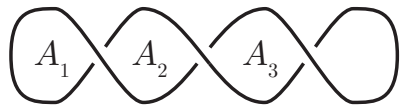

(c)

Figure 2. There is no negative slice of a flat-atinfinity planar Lagrangian with diagram (a) $8^{-}(A)$, (b) $C^{-+-}\left(A_{1}, A_{2}, A_{3}\right)$, or (c) $C^{---}\left(A_{1}, A_{2}, A_{3}\right)$. 
(1) For any $\mathbf{S} \in \mathcal{S},[\emptyset] \unlhd \mathbf{S}$.

(2) There exist areas $A_{1}, A_{2}, B_{1}, B_{2}, B_{3}, C$ so that

$$
\left[\widetilde{8}^{+}\left(A_{1}\right)+\widetilde{8}^{+}\left(A_{2}\right)\right] \triangleleft\left[\widetilde{C}^{+-+}\left(B_{1}, B_{2}, B_{3}\right)\right] \triangleleft\left[\widetilde{8}^{+}(C)\right] .
$$

(3) There exists areas $A_{1}, A_{2}, A_{3}, B_{1}, B_{2}, C$ so that

$$
\left[\widetilde{8}^{-}\left(A_{1}, A_{2}\right) \times \widetilde{8}^{+}\left(A_{3}\right)\right] \triangleleft\left[\widetilde{8}^{-}\left(B_{1}\right) \odot \widetilde{8}^{+}\left(B_{2}\right)\right] \triangleleft\left[\widetilde{8}^{+}(C)\right] .
$$

See Section 3 for a verification of these examples.

Unlike in Arnold's original construction, the cobordisms defining $\unlhd$ are directed, and hence do not give rise to an equivalence relation. In fact, on the subset $\mathcal{S}_{c} \subset \mathcal{S}$ of connected slices, we can show that the cobordisms defining $\unlhd$ give rise to a partial order:

Theorem 1.4. Using the notation of Figure 1,

(1) The relation $\unlhd$ is not symmetric: in particular, for all $0<A<B$,

$$
\left[\widetilde{8}^{+}(A)\right] \unlhd\left[\widetilde{8}^{+}(B)\right] \text {, while }\left[\widetilde{8}^{+}(B)\right] \unlhd\left[\widetilde{8}^{+}(A)\right] .
$$

(2) Not all slices are related: in particular, for any $A, B>0$,

$$
\left[\widetilde{8}^{+}(A)\right] \unlhd\left[\widetilde{C}^{(+,-,+)}(A, B, B)\right] \text {, and }\left[\widetilde{C}^{(+,-,+)}(A, B, B)\right] \unlhd\left[\widetilde{8}^{+}(A)\right] \text {. }
$$

(3) The relation $\unlhd$ gives $\mathcal{S}_{c}$ the structure of a partially ordered set.

The second part of the theorem implies that $\mathcal{S}_{c}$ is not totally ordered by $\unlhd$. We believe that the relation $\unlhd$ gives the entire set $\mathcal{S}$ the structure of a partially ordered set. However, the obstructions used in the proof of antisymmetry work only with flat-at-infinity planar Lagrangians, and the gluing operations used in the proof of transitivity and antisymmetry may, in general, take us outside that set.

Further structure on $\mathcal{S}$ is suggested by the second part of Example 1.3: the curve $\widetilde{8}^{+}\left(A_{1}\right)+\widetilde{8}^{+}\left(A_{2}\right)$ can be thought of as a sum of slices. In fact, $\mathcal{S}$ becomes a commutative monoid when addition is defined by disjoint union:

Definition 1.5. For $\mathbf{S}, \mathbf{S}^{\prime} \in \mathcal{S}$, let $S \in \mathbf{S}, S^{\prime} \in \mathbf{S}^{\prime}, S \subset\left\{x_{1}<0\right\}$, and $S^{\prime} \subset\left\{x_{1}>0\right\}$. Define:

$$
\mathbf{S}+\mathbf{S}^{\prime}=\left[S \cup S^{\prime}\right]
$$

That this addition is well-defined on $\mathcal{S}$ will be proven below in Section 2 . The additive structure has an interesting interaction with the relations $\unlhd$ and $\triangleleft$ :

Theorem 1.6. (1) The relation $\unlhd$ is not compatible with + : in particular,

$[\emptyset] \unlhd\left[\widetilde{8}^{+}(A)\right]$ and $\left[\widetilde{8}^{+}(A)\right] \unlhd\left[\widetilde{8}^{+}(A)\right]$, but $\left[\widetilde{8}^{+}(A)\right] \unlhd\left[\widetilde{8}^{+}(A)\right]+\left[\widetilde{8}^{+}(A)\right]$.

(2) The strict relation $\triangleleft$ is compatible with + : for all $\mathbf{S}, \mathbf{S}^{\prime}, \mathbf{T}, \mathbf{T}^{\prime} \in \mathcal{S}$, if $\mathbf{S} \triangleleft \mathbf{S}^{\prime}$ and $\mathbf{T} \triangleleft \mathbf{T}^{\prime}$ then $\mathbf{S}+\mathbf{T} \triangleleft \mathbf{S}^{\prime}+\mathbf{T}^{\prime}$. 
The rest of the paper is structured as follows: in Section 2, we verify that $\unlhd, \triangleleft$, and + are well-defined on $\mathcal{S}$; we also prove part (2) of Theorem 1.6. Section 3 describes constructions of the examples in Figure 1. Section 4 discusses the machinery of slice capacities, a concept introduced in [7], in

order to provide obstructions to certain curves being realized as elements of $\widetilde{\mathcal{S}}$ and to show that certain relations between elements of $\mathcal{S}$ do not exist. These capacities, influenced greatly by ideas of Viterbo [8], are defined through the theory of generating families, and it is for this reason that we restrict ourselves to flat-at-infinity planar Lagrangians. Calculations of capacities prove parts (1) and (2) of Theorem 1.4 and part (1) of Theorem 1.6. Finally, Section 5 contains the proof of part (3) of Theorem 1.4, namely that $\unlhd$ is a partial order on the set of connected slices $\mathcal{S}_{c}$.

Acknowledgments. We thank V.I. Arnold for his many inspirational ideas and beautiful results over the years.

\section{Relations And Operations}

In this section, we will verify that the relation $\triangleleft$ and the operation + are well-defined with respect to the equivalence relation $\sim$ on $\widetilde{\mathcal{S}}$.

2.1. The Definition of $\triangleleft$ and $\unlhd$. We first show that the relation $\triangleleft$ (and $\unlhd)$ is well-defined with respect to the equivalence on the set of slices.

Proof of Proposition 1.2. Suppose that $S_{0} \triangleleft T_{0}$ via the flat-at-infinity planar Lagrangian $L$ at levels $a<b<0$. Suppose further that $S_{0} \sim S_{1}$ (resp. $T_{0} \sim T_{1}$ ) via a diffeomorphism generated by the Hamiltonian function $H_{t}\left(x_{1}, y_{1}\right)$ (resp. $\left.G_{t}\left(x_{1}, y_{1}\right)\right)$; here we are using the fact that the areapreserving diffeomorphisms guaranteed by $\sim$ are necessarily Hamiltonian. Choose interval neighborhoods $U_{a}$ and $U_{b}$ of $a, b \in \mathbb{R}$ so that $U_{a} \cap U_{b}=\emptyset$ and $0 \notin U_{b}$; choose smaller neighborhoods $V_{a} \subset U_{a}$ and $V_{b} \subset V_{b}$ of $a$ and $b$. Then let $\sigma: \mathbb{R} \rightarrow \mathbb{R}$ be a smooth function that is equal to 1 on $V_{a}$ and to 0 outside $U_{a}$. Similarly, let $\tau: \mathbb{R} \rightarrow \mathbb{R}$ be equal to 1 on $V_{b}$ and to 0 outside $U_{b}$. It is then straightforward to verify that

$$
\mathbf{H}_{t}\left(x_{1}, x_{2}, y_{1}, y_{2}\right)=\sigma\left(y_{2}\right) H_{t}\left(x_{1}, y_{1}\right)+\tau\left(y_{2}\right) G_{t}\left(x_{1}, y_{1}\right)
$$

generates a Hamiltonian isotopy of $\mathbb{R}^{4}$ taking $L$ to a flat-at-infinity planar Lagrangian joining $S_{1}$ to $T_{1}$.

2.2. The Definition of + . To show that the sum $\mathbf{S}+\mathbf{S}^{\prime}$ is well-defined, we will prove the following proposition:

Proposition 2.1. $\quad$ (1) The sum $\mathbf{S}+\mathbf{S}^{\prime}$ does not depend upon the choice of representatives of $\mathbf{S}$ in $\left\{x_{1}<0\right\}$ or of $\mathbf{S}^{\prime}$ in $\left\{x_{1}>0\right\}$.

(2) The sum of two elements of $\mathcal{S}$ is again an element of $\mathcal{S}$. 
Proof of Part (1). Suppose that $S$ and $\bar{S}$ are both representatives of $\mathbf{S}$ in $\left\{x_{1}<0\right\}$; it suffices to show that $S \cup S^{\prime} \sim \bar{S} \cup S^{\prime}$. By hypothesis, there exists a compactly supported area-preserving isotopy $\varphi_{t}$ of the $x_{1} y_{1}$-plane so that $\left(\varphi_{1} \times \mathrm{id}\right)(S)=\bar{S}$. We construct a compactly supported area-preserving isotopy $\widetilde{\varphi}_{t}$ of $\left\{x_{1}<0\right\}$ so that $\left(\widetilde{\varphi}_{1} \times \mathrm{id}\right)(S)=\bar{S}$ as follows. Choose $\epsilon>0$ so that $S, \bar{S} \subset\left\{x_{1}<-\epsilon\right\}$. There exists an area-preserving diffeomorphism $\tau$ so that $\tau\left(\left\{x_{1}<0\right\}\right)=\mathbb{R}^{2}$ and $\tau$ is the identity on $\left\{x_{1} \leq-\epsilon\right\}$. Then $\tau^{-1} \circ \varphi_{t} \circ \tau$ is a compactly supported area-preserving isotopy of $\left\{x_{1}<0\right\}$ that extends by the identity to a compactly supported area-preserving isotopy $\widetilde{\varphi}_{t}$ of $\mathbb{R}^{2}$ with the property that $\left(\widetilde{\varphi}_{1} \times\right.$ id $)\left(S \cup S^{\prime}\right)=\left(\bar{S} \cup S^{\prime}\right)$.

The proof of the second part of the proposition - that the sum of two elements of $\mathcal{S}$ is again an element of $\mathcal{S}$ - relies upon two constructions. The first is that there is a Hamiltonian diffeomorphism that can shift the level of slices:

Lemma 2.2. For any $a<b<0$, there is a Hamiltonian diffeomorphism $\psi$ so that $\psi$ is the identity on $\left\{y_{2} \geq b\right\}$, and on $\left\{y_{2} \leq a\right\}, \psi$ is a translation by $m$ in the $y_{2}$-direction for any $m<b-a$.

Proof. Consider the Hamiltonian $H\left(x_{1}, x_{2}, y_{1}, y_{2}\right)=\sigma\left(y_{2}\right) x_{2}$, where $\sigma\left(y_{2}\right)$ is a smooth function such that

$$
\sigma\left(y_{2}\right)= \begin{cases}0, & y_{2} \geq b \\ m, & y_{2} \leq \max \{a, a+m\}\end{cases}
$$

It is easy to verify that $H$ generates an integrable vector field that gives the desired $\psi$.

The second construction is that of a "connect sum" for flat-at-infinity planar Lagrangians.

Definition 2.3. Given two flat-at-infinity planar Lagrangians $L, L^{\prime}$, a connect sum of $L$ and $L^{\prime}$, denoted $L \# L^{\prime}$ is defined as follows. Assume $L$ (resp. $L^{\prime}$ ) agrees with the zero-section $L_{0}$ outside a compact set $K$ (resp. $K^{\prime}$ ) of $\mathbb{R}^{4}$. Choose $x_{1}$-translations $\tau$ of $L$ and $\tau^{\prime}$ of $L^{\prime}$ so that $\tau(K) \subset\left\{x_{1}<0\right\}$ and $\tau^{\prime}\left(K^{\prime}\right) \subset\left\{x_{1}>0\right\}$. Then $L \# L^{\prime}$ is defined to be

$$
\left(\tau(L) \cap\left\{x_{1} \leq 0\right\}\right) \cup\left(\tau^{\prime}\left(L^{\prime}\right) \cap\left\{x_{1} \geq 0\right\}\right) .
$$

The following lemma shows that, up to Hamiltonian isotopy, the construction of the connect sum does not depend on $\tau$ and $\tau^{\prime}$.

Lemma 2.4. Let $L \# L^{\prime}$ and $L \tilde{\#} L^{\prime}$ be two connect sums with respect to $x_{1}$-translations $\tau, \tau^{\prime}$ and $\tilde{\tau}, \tilde{\tau}^{\prime}$. Then there exists a compactly supported areapreserving isotopy $\varphi_{t}$ of the $x_{1} y_{1}$-plane so that $\left(\varphi_{1} \times \mathrm{id}\right)\left(L \# L^{\prime}\right)=L \tilde{\#} L^{\prime}$.

Proof. Choose sets $U, U^{\prime}$ in the $x_{1} y_{1}$-plane containing the projections of $K, K^{\prime}$ of Definition 2.3. There exists a compactly supported area-preserving isotopy $\varphi_{t}$ translating $\tau(U)$ to $\tilde{\tau}(U)$ and $\tau^{\prime}\left(U^{\prime}\right)$ to $\tilde{\tau}^{\prime}\left(U^{\prime}\right)$ that preserves $\left\{y_{1}=0\right\}$. It follows that $\left(\varphi_{1} \times \mathrm{id}\right)\left(L \# L^{\prime}\right)=L \tilde{\#} L^{\prime}$, as desired. 
With these two constructions, we can prove the second part of the proposition:

Proof of Proposition 2.1(2). Suppose that $S \in \mathbf{S}, S \subset\left\{x_{1}<0\right\}, i_{a}(S)=$ $L_{a}$, and that $S^{\prime} \in \mathbf{S}^{\prime}, S^{\prime} \subset\left\{x_{1}>0\right\}$, and $i_{b}\left(S^{\prime}\right)=L_{b}^{\prime}$. By Lemma 2.2, we may assume that $a=b$. At the cost of passing to equivalent slices using the compactly supported translation construction from the proof of part (1), we may then conclude that $\left(L \# L^{\prime}\right)_{a}$ is a representative of $\mathbf{S}+\mathbf{S}^{\prime}$.

2.3. Proof of Theorem 1.6(2). We begin by generalizing the level-shifting lemma:

Lemma 2.5. Consider a flat-at-infinity planar Lagrangian $L$ and its slices $L_{a}, L_{b}$ for some $a<b<0$. Then for all $c<d<0$, there exists a flat-atinfinity planar Lagrangian $M$ so that $L_{a} \sim M_{c}$ and $L_{b} \sim M_{d}$.

Proof. $M$ is obtained by applying a composition of level-preserving Hamiltonian diffeomorphisms as in Lemma 2.2 to $L$.

We can now easily prove that the strict relation $\triangleleft$ is compatible with + :

Proof of Theorem 1.6(2). We know there exist flat-at-infinity planar Lagrangians $L$ and $M$ and representatives $S \in \mathbf{S}$, etc., such that for some $a<b<0$ and $c<d<0$,

$$
\begin{aligned}
i_{b}\left(S^{\prime}\right) & =L_{b} & i_{d}\left(T^{\prime}\right) & =M_{d} \\
i_{a}(S) & =L_{a} & i_{c}(T) & =M_{c} .
\end{aligned}
$$

By Lemma 2.5, we may assume that $a=c$ and $b=d$. Then the connect sum $L \# M$ gives a flat-at-infinity planar Lagrangian with $\left[(L \# M)_{a}\right]=\mathbf{S}+\mathbf{T}$ and $\left[(L \# M)_{b}\right]=\mathbf{S}^{\prime}+\mathbf{T}^{\prime}$.

\section{Constructions of Slices and Cobordisms}

Figure 1 gives examples of $x_{1} y_{1}$-diagrams of negative slices. The unknotted figure- 8 diagram with a positive self-crossing bounding two lobes of area $A$ shown in Figure $1\left(\right.$ a) will be denoted by $8^{+}(A)$. Choose $A_{1}, A_{2}, A_{3}$ so that $A_{1}-A_{2}+A_{3}>0$. Then the "caterpillar" with three crossings of signs ,,+-+ and four lobes of areas $A_{1}, A_{2}, A_{3}$, and $A_{4}=A_{1}-A_{2}+A_{3}$ shown in (b) will be denoted by $C^{+-+}\left(A_{1}, A_{2}, A_{3}\right)$. The two component sum of unknotted figure-8's with positive crossings shown in (c) will be denoted by $8^{+}\left(A_{1}\right)+8^{+}\left(A_{2}\right)$. The figure-8 "inside" another figure- 8 shown in $(\mathrm{d})$ will be denoted by $8^{-}\left(A_{1}\right) \odot 8^{+}\left(A_{2}\right)$. Lastly, the "merged" figure-8's shown in

(e) will be denoted by $8^{-}\left(A_{1}, A_{2}\right) \times 8^{+}\left(A_{3}\right)$.

That each of these curves is the diagram of a negative slice of an embedded, flat-at-infinity planar Lagrangian may be proven by examining the graph of $d F$ for an explicit function $F: \mathbb{R}^{2} \rightarrow \mathbb{R}$. In [7], an explicit generating family is constructed to generate the curve in (a), and modifications of this construction can be used to generate many of the specified slices. Other slices were found through computer-aided calculations; the first two 
authors wrote programs in Mathematica to explore the shapes that can be realized as slices and to see how the slices evolve. ${ }^{1}$ For example, in Figure 1, explicit calculations show that the lifts of the top figure- 8 diagram together with lifts of the diagrams in each of the two columns represent sequences of related slices:

(1) There exist areas $A_{1}, A_{2}, B_{1}, B_{2}, B_{3}, C$ so that

$$
\left[\widetilde{8}^{+}\left(A_{1}\right)+\widetilde{8}^{+}\left(A_{2}\right)\right] \triangleleft\left[\widetilde{C}^{+-+}\left(B_{1}, B_{2}, B_{3}\right)\right] \triangleleft\left[\widetilde{8}^{+}(C)\right] ;
$$

(2) There exist areas $A_{1}, A_{2}, A_{3}, B_{1}, B_{2}, C$ so that

$$
\left[\widetilde{8}^{-}\left(A_{1}, A_{2}\right) \times \widetilde{8}^{+}\left(A_{3}\right)\right] \triangleleft\left[\widetilde{8}^{-}\left(B_{1}\right) \odot \widetilde{8}^{+}\left(B_{2}\right)\right] \triangleleft\left[\widetilde{8}^{+}(C)\right] .
$$

\section{Obstructions to Slices and Cobordisms}

In order to show that a given curve cannot appear as a negative slice, or to show that two slices are not related, we will employ the slice capacity machinery developed in [7]. In particular, we will be able to prove that the curves in Figure 2 cannot be diagrams of any element of $\widetilde{\mathcal{S}}$. We will also prove parts (1) and (2) of Theorem 1.4 and part (1) of Theorem 1.6.

4.1. Capacities. For each slice $L_{a}$ of a flat-at-infinity planar Lagrangian $L$ at a generic height $a$, we define two lower and two upper capacities:

$$
c_{ \pm}^{L, a}: H^{*}\left(L_{a}\right) \rightarrow(-\infty, 0], \quad C_{ \pm}^{L, a}: H^{*}\left(L_{a}\right) \rightarrow[0, \infty) .
$$

Below is a brief description of the construction of these capacities from the theory of generating families. Full details and citations can be found in [7]; the original constructions that inspired these capacities appear in Viterbo's paper [8].

If $L \subset \mathbb{R}^{4}$ is a flat-at-infinity planar Lagrangian then there is a quadraticat-infinity generating family $F: \mathbb{R}^{2} \times \mathbb{R}^{N} \rightarrow \mathbb{R}$ for $L$. In particular,

$$
L=\left\{\left(x_{1}, x_{2}, \frac{\partial F}{\partial x_{1}}\left(x_{1}, x_{2}, \mathbf{e}\right), \frac{\partial F}{\partial x_{2}}\left(x_{1}, x_{2}, \mathbf{e}\right)\right): \frac{\partial F}{\partial \mathbf{e}}=0\right\} .
$$

Moreover, this quadratic-at-infinity generating family is unique up to addition of a constant, fiber-preserving diffeomorphism, and stabilization. To study a slice $L_{a}$ of $L$, we consider the difference function

$$
\Delta_{a}: \mathbb{R} \times \mathbb{R}^{1+N} \times \mathbb{R}^{1+N} \rightarrow \mathbb{R}
$$

defined by:

$$
\Delta_{a}\left(x_{1}, x_{2}, \mathbf{e}, \tilde{x}_{2}, \tilde{\mathbf{e}}\right)=F\left(x_{1}, x_{2}, \mathbf{e}\right)-F\left(x_{1}, \tilde{x}_{2}, \tilde{\mathbf{e}}\right)-a\left(x_{2}-\tilde{x}_{2}\right) .
$$

The difference function is Morse-Bott. Its critical points are of two types:

\footnotetext{
${ }^{1}$ The programs are user-friendly and can be found in a web appendix to this paper located in the "Research" section of the web page

http: //www.haverford.edu/math/jsabloff

We invite readers to use and experiment with these programs.
} 
(1) For each double point $\left(x_{1}, y_{1}\right)$ of $\pi\left(L_{a}\right) \subset \mathbb{R}^{2}$, there are two nondegenerate critical points $\left(x_{1}, x_{2}, \mathbf{e}, \tilde{x}_{2}, \tilde{\mathbf{e}}\right)$ and $\left(x_{1}, \tilde{x}_{2}, \tilde{\mathbf{e}}, x_{2}, \mathbf{e}\right)$ whose critical values are either both 0 or are $\pm v$, for some $v \neq 0$.

(2) A non-degenerate critical submanifold diffeomorphic to $L_{a}$ with critical value 0 and index $1+N$.

The critical value and index of a critical point $\left(x_{1}, x_{2}, \mathbf{e}, \tilde{x}_{2}, \tilde{\mathbf{e}}\right)$ corresponding to a crossing between two branches of the same component of $L_{a}$ may be calculated from a diagram of $L_{a}$. Choose a "capping path" $\gamma$ in $L_{a}$ that starts at the image of $\left(x_{1}, \tilde{x}_{2}, \tilde{\mathbf{e}}\right)$ and ends at the image of $\left(x_{1}, x_{2}, \mathbf{e}\right)$. The critical value is the negative signed area of the region bounded by $\gamma$, and the index is equal to $N+1-\mu(\bar{\Gamma})$, where $\bar{\Gamma}$ is the closure of the loop of subspaces $T \gamma(t)$ by a clockwise rotation and $\mu$ is the Maslov index; see Section 6 of $[7]$.

The distinction between positive and negative capacities comes from a splitting of the domain $\mathbb{R} \times \mathbb{R}^{1+N} \times \mathbb{R}^{1+N}$ into positive and negative pieces:

$\left.\mathcal{P}_{+}=\left\{x_{1}, x_{2}, \mathbf{e}, \tilde{x}_{2}, \tilde{\mathbf{e}}\right): x_{2} \leq \tilde{x}_{2}\right\}, \quad \mathcal{P}_{-}=\left\{\left(x_{1}, x_{2}, \mathbf{e}, \tilde{x}_{2}, \tilde{\mathbf{e}}\right): x_{2} \geq \tilde{x}_{2}\right\}$.

Denote the sublevel sets of $\Delta_{a}$ by $\Delta_{a}^{\lambda}$, and then define

$$
\Delta_{a, \pm}^{\lambda}=\Delta_{a}^{\lambda} \cap \mathcal{P}_{ \pm}
$$

For $\eta>0$ chosen so that 0 is the only critical value of $\Delta_{a}$ in $[-\eta, \eta]$, the following maps may be defined:

$$
\begin{aligned}
& \varphi_{a, \pm}^{\lambda}: H^{k}\left(L_{a}\right) \rightarrow H^{k+N+1}\left(\Delta_{a, \pm}^{\eta}, \Delta_{a, \pm}^{\lambda}\right), \quad \text { and } \\
& \Phi_{a, \pm}^{\Lambda}: H^{k}\left(L_{a}\right) \rightarrow H^{k+N+2}\left(\Delta_{a, \pm}^{\Lambda}, \Delta_{a, \pm}^{\eta}\right) .
\end{aligned}
$$

The first is the composition of the Thom isomorphism, the map

$$
p: H^{k+N+1}\left(\Delta_{a}^{\eta}, \Delta_{a}^{-\eta}\right) \rightarrow H^{k+N+1}\left(\Delta_{a}^{\eta}, \Delta_{a}^{\lambda}\right)
$$

in the exact sequence of the triple $\left(\Delta_{a}^{\eta}, \Delta_{a}^{-\eta}, \Delta_{a}^{\lambda}\right)$, and a map in a MayerVietoris sequence relating $\Delta_{a}^{\lambda}$ to $\Delta_{a, \pm}^{\lambda}$. The second map is similar, with the connecting homomorphism of the exact sequence of the triple $\left(\Delta_{a}^{\Lambda}, \Delta_{a}^{\eta}, \Delta_{a}^{-\eta}\right)$ replacing $p$.

The lower and upper capacities are then defined to be:

$$
\begin{gathered}
c_{ \pm}^{L, a}(u)=\sup \left\{\lambda<0: \varphi_{a, \pm}^{\lambda}(u)=0\right\} \\
C_{ \pm}^{L, a}(u)=\inf \left\{\Lambda>0: \Phi_{a, \pm}^{\Lambda}(u) \neq 0\right\} .
\end{gathered}
$$

In both cases, if the set is empty then the capacity is 0 . The capacities $c_{ \pm}^{L, a}(u)$ and $C_{ \pm}^{L, a}(u)$ are critical values of $\Delta_{a}$ and are independent of the generating family $F$ used to define $L$. 
4.2. Generalities on the Computation of Capacities. In this paper, we use the following three properties of the capacities:

(1) (Invariance) If $L^{0}$ and $L^{1}$ are flat-at-infinity planar Lagrangians that are isotopic via a compactly supported Hamiltonian isotopy that sends the hyperplane $\left\{y_{2}=a\right\}$ to itself then $c^{L^{0}, a}(u)=c^{L^{1}, a}(u)$, for any of the four capacities and any cohomology class $u .^{2}$

(2) (Monotonicity) Suppose $a<b<0$, and $a$ and $b$ are generic heights of a flat-at-infinity planar Lagrangian $L \subset \mathbb{R}^{4}$. Let $W=\bigcup_{t \in[a, b]} L_{t}$ be the cobordism between $L_{a}$ and $L_{b}$ given by $L$, and let $j_{t}: L_{t} \rightarrow W$ be the inclusion map. If $u \in H^{*}(W)$ then:

$$
\begin{array}{rlrl}
c_{+}^{L, a}\left(j_{a}^{*} u\right) & \leq c_{+}^{L, b}\left(j_{b}^{*} u\right), & C_{+}^{L, a}\left(j_{a}^{*} u\right), & \leq C_{+}^{L, b}\left(j_{b}^{*} u\right), \\
c_{-}^{L, a}\left(j_{a}^{*} u\right) & \geq c_{-}^{L, b}\left(j_{b}^{*} u\right), & C_{-}^{L, a}\left(j_{a}^{*} u\right) \geq C_{-}^{L, b}\left(j_{b}^{*} u\right) .
\end{array}
$$

If at least one of the capacities in an inequality above is nonzero, the inequality is strict.

(3) (Non-Vanishing) For any generic, nonempty slice $L_{a}$ of a flat-atinfinity planar Lagrangian $L \subset \mathbb{R}^{4}$ and for any nonzero $u \in H^{*}\left(L_{a}\right)$, at least one of the four capacities $c_{ \pm}^{L, a}(u), C_{ \pm}^{L, a}(u)$ is nonzero.

Although the capacities for a slice depend on the entire Lagrangian, it is sometimes possible to compute these numbers only knowing the slice $L_{a}$. The capacities always lie at critical values of the difference function $\Delta_{a}$, so their calculation relies on the computation of critical values and indices of critical points of $\Delta_{a}$ and, in more complicated situations, on examination of the exact sequences used to define $\varphi_{a, \pm}^{\lambda}$ and $\Phi_{a, \pm}^{\Lambda}$. A foundational computation is the following:

Theorem 4.1 ([7]). $\quad$ (1) If $L$ is any flat-at-infinity planar Lagrangian with slice $L_{a}$ having diagram $8^{-}(A)$ then, for $0 \neq u \in H^{0}\left(L_{a}\right)$ and $0 \neq v \in H^{1}\left(L_{a}\right)$,

$c_{+}^{L, a}(u)=-A, \quad c_{-}^{L, a}(u)=0, \quad C_{+}^{L, a}(v)=0, \quad C_{-}^{L, a}(v)=A$.

(2) If $L$ is any flat-at-infinity planar Lagrangian with slice $L_{a}$ having diagram $8^{+}(A)$ then, for $0 \neq u \in H^{0}\left(L_{a}\right)$ and $0 \neq v \in H^{1}\left(L_{a}\right)$, $c_{+}^{L, a}(u)=0, \quad c_{-}^{L, a}(u)=-A, \quad C_{+}^{L, a}(v)=A, \quad C_{-}^{L, a}(v)=0$.

Implicit in the proof of the theorem above is the following general computational principle:

Lemma 4.2. Suppose that $u \in H^{k}\left(L_{a}\right)$. If there is no critical point of $\Delta_{a}$ in $\mathcal{P}_{ \pm}$of index $k+N$ (resp. $k+N+2$ ) and negative (resp. positive) critical value then $c_{ \pm}^{L, a}(u)=0$ (resp. $\left.C_{ \pm}^{L, a}(u)=0\right)$.

Another useful lemma is:

\footnotetext{
${ }^{2}$ This is slightly different from the statement of invariance in [7], but the proof is exactly the same.
} 
Lemma 4.3. Let $a<0$ be a generic height of a flat-at-infinity planar Lagrangian $L$, and let $j_{a}: L_{a} \rightarrow L$ be the inclusion map. For any $u \in H^{*}(L)$, $c_{+}^{L, a}\left(j_{a}^{*} u\right)=0$ and $C_{-}^{L, a}\left(j_{a}^{*} u\right)=0$.

Proof. Since $L$ is flat-at-infinity, there exists $a^{\prime}<a$ so that $L_{a^{\prime}}=\emptyset$. Since $c_{+}^{L, a^{\prime}}\left(j_{a^{\prime}}^{*} u\right)$ and $C_{-}^{L, a^{\prime}}\left(j_{a^{\prime}}^{*} u\right)$ vanish, Monotonicity and the definition of the capacities imply that:

$$
\begin{aligned}
& 0=c_{+}^{L, a^{\prime}}\left(j_{a^{\prime}}^{*} u\right) \leq c_{+}^{L, a}\left(j_{a}^{*} u\right) \leq 0, \\
& 0=C_{-}^{L, a^{\prime}}\left(j_{a^{\prime}}^{*} u\right) \geq C_{-}^{L, a}\left(j_{a}^{*} u\right) \geq 0 .
\end{aligned}
$$

4.3. Obstructions to the Existence of Slices. We begin our exploration of the use of capacities as obstructions by showing that certain curves cannot appear as negative slices of flat-at-infinity planar Lagrangians. The simplest example is that $8^{-}(A)$ cannot be the diagram of a negative slice; this follows immediately from Theorem 4.1 and Lemma 4.3.

More interestingly, some slight modifications of the realizable caterpillar diagram in Figure 1(b) cannot be the diagram of a negative slice.

Proposition 4.4. Consider $A_{1}, A_{2}, A_{3}$ so that $A_{1}-A_{2}+A_{3}>0$. Then neither of the curves $C^{- \pm-}\left(A_{1}, A_{2}, A_{3}\right)$ pictured in Figure $2(b, c)$ can be the diagram of a generic negative slice of any flat-at-infinity planar Lagrangian.

Proof. Suppose $C^{- \pm-}\left(A_{1}, A_{2}, A_{3}\right)$ is the diagram of a negative slice of the flat-at-infinity planar Lagrangian $L$. We will show that $c_{-}^{L, a}(u)=0$ and $C_{+}^{L, a}(u)=0$, for all $u \in H^{0}\left(L_{a}\right)$. This, together with Lemma 4.3 , gives a contradiction to the Non-Vanishing property of capacities.

The difference function $\Delta_{a}$ associated to $L$ has six non-degenerate critical points: $q_{ \pm}^{1}$ come from the leftmost crossing, $q_{ \pm}^{2}$ come from the center crossing, and $q_{ \pm}^{3}$ come from the rightmost crossing. Using the capping paths described in the previous section, we find the following indices, critical values, and $\mathcal{P}_{ \pm}$locations for the critical points of $C^{-+-}\left(A_{1}, A_{2}, A_{3}\right)$ :

\begin{tabular}{c|ccc} 
& Location & Index & Critical Value \\
\hline$q_{-}^{1}$ & $\mathcal{P}_{-}$ & $N+3$ & $A_{1}$ \\
$q_{+}^{1}$ & $\mathcal{P}_{+}$ & $N$ & $-A_{1}$ \\
$q_{-}^{2}$ & $\mathcal{P}_{-}$ & $N+2$ & $A_{1}-A_{2}$ \\
$q_{+}^{2}$ & $\mathcal{P}_{+}$ & $N+1$ & $A_{2}-A_{1}$ \\
$q_{-}^{3}$ & $\mathcal{P}_{-}$ & $N+3$ & $A_{1}-A_{2}+A_{3}$ \\
$q_{+}^{3}$ & $\mathcal{P}_{+}$ & $N$ & $-A_{1}+A_{2}-A_{3}$
\end{tabular}

The critical points of $C^{---}$have similar properties, except that the indices and critical values of $q_{ \pm}^{2}$ are swapped.

Suppose $u \in H^{0}\left(L_{a}\right)$. For any $C^{- \pm-}\left(A_{1}, A_{2}, A_{3}\right)$, since both critical points of index $N$ live in $\mathcal{P}_{+}$, it follows from Lemma 4.2 that $c_{-}^{L, a}(u)=0$. 


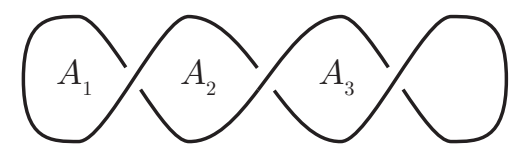

Figure 3. Can the curve $C^{+++}\left(A_{1}, A_{2}, A_{3}\right)$ be realized as a generic negative slice of some flat-at-infinity planar Lagrangian?

Further, for all curves of the form $C^{-+-}$, the only non-degenerate critical point of index $N+2$ lives in $\mathcal{P}_{-}$, and thus it follows that $C_{+}^{L, a}(u)=0$.

It remains to show that for all curves of the form $C^{---}$, we have $C_{+}^{L, a}(u)=$ 0 . First consider the case where $A_{1} \leq A_{2}$. In this case, the critical point $q_{-}^{2}$ has critical value $A_{1}-A_{2} \leq 0$, so it follows from Lemma 4.2 that $C_{+}^{L, a}(u)=0$. In the case where $A_{1}>A_{2}$, the critical point $q_{-}^{2}$ has critical value $A_{1}-A_{2}>0$, so a more sophisticated argument is required to show that $\Phi_{a,+}^{\Lambda}(u)=0$, for all $\Lambda>0$. Suppose there exists $\Lambda>0$ so that $\Phi_{a,+}^{\Lambda}(u) \neq 0$. We may assume that $\Lambda$ lies between the critical values associated to the points of index $N+2$ and $N+3$ :

$$
A_{1}-A_{2}<\Lambda<\min \left\{A_{1}, A_{1}-A_{2}+A_{3}\right\} .
$$

By examining a long exact sequence associated to $\left(\Delta_{a}^{\Lambda}, \Delta_{a}^{\eta}, \Delta_{a}^{-\eta}\right)$, we see that the assumption that $\Phi_{a,+}^{\Lambda}(u) \neq 0$ implies:

$$
\operatorname{rank} H^{N+2}\left(\Delta_{a}^{\Lambda}, \Delta_{a}^{-\eta}\right)=\operatorname{rank} H^{N+2}\left(\Delta_{a}^{\eta}, \Delta_{a}^{-\eta}\right)=1 .
$$

Next, we examine the long exact sequence associated to $\left(\Delta_{a}^{\theta}, \Delta_{a}^{-\eta}, \Delta_{a}^{-\theta}\right)$, for some $\theta \gg 0$. The fact that $H^{*}\left(\Delta_{a}^{\theta}, \Delta_{a}^{-\theta}\right)=0$ (see Lemma 5.3 of [7]) implies that:

$$
H^{N+3}\left(\Delta_{a}^{\theta}, \Delta_{a}^{-\eta}\right) \simeq H^{N+2}\left(\Delta_{a}^{-\eta}, \Delta_{a}^{-\theta}\right)=0 .
$$

Finally, using (4.7) in the long exact sequence associated to the triple $\left(\Delta_{a}^{\theta}, \Delta_{a}^{\Lambda}, \Delta_{a}^{-\eta}\right)$, we find that there is a surjective map from $H^{N+2}\left(\Delta_{a}^{\Lambda}, \Delta_{a}^{-\eta}\right)$ to $H^{N+3}\left(\Delta_{a}^{\theta}, \Delta_{a}^{\Lambda}\right)$. However, the indices and critical values of the critical points of $\Delta_{a}$ imply that for $\theta \gg 0$, we have $\operatorname{rank} H^{N+3}\left(\Delta_{a}^{\theta}, \Delta_{a}^{\Lambda}\right)=2$. Thus, we obtain a surjective map from a group of rank 1 (by (4.6)) to a group of rank 2, an impossibility. It follows that $\Phi_{a,+}^{\Lambda}(u)=0$, for all $\Lambda$, and hence $C_{+}^{L, a}(u)=0$, as desired.

In contrast to the definitive statements above about caterpillars, we do not know if the caterpillar $C^{+++}\left(A_{1}, A_{2}, A_{3}\right)$ in Figure 3 can be realized as the diagram of a generic negative slice of a flat-at-infinity planar Lagrangian. We have not been able to reproduce it using our computer-aided calculations, but capacity arguments as in the proof of Proposition 4.4 do not rule out its possibility.

Capacities of slices of Lagrangians that are connect sums can easily be calculated in terms of the capacities of the pieces: 

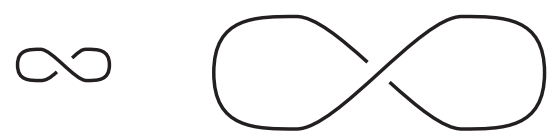

Figure 4. This pair of curves $8^{-}(A)+8^{+}(B)$ cannot be realized as the diagram of a negative slice of any connect sum of flat-at-infinity planar Lagrangians. Can it be realized as a negative slice of some flat-at-infinity planar Lagrangian?

Lemma 4.5. For $\left(u_{1}, u_{2}\right) \in H^{0}\left(\left(L_{1} \# L_{2}\right)_{a}\right)=H^{0}\left(\left(L_{1}\right)_{a}\right) \oplus H^{0}\left(\left(L_{2}\right)_{a}\right)$,

$$
\begin{array}{r}
c_{ \pm}^{L_{1} \# L_{2}, a}\left(u_{1}, 0\right)=c_{ \pm}^{L_{1}, a}\left(u_{1}\right), \quad c_{ \pm}^{L_{1} \# L_{2}, a}\left(0, u_{2}\right)=c_{ \pm}^{L_{2}, a}\left(u_{2}\right), \\
\text { and } c_{ \pm}^{L_{1} \# L_{2}, a}\left(u_{1}, u_{2}\right)=\max \left\{c_{ \pm}^{L_{1}, a}\left(u_{1}\right), c_{ \pm}^{L_{2}, a}\left(u_{2}\right)\right\} .
\end{array}
$$

Proof. By Lemma 2.4 and Invariance, it suffices to work with a connect sum obtained by choosing the compact sets $K_{i}$ in Definition 2.3 quite large. Using stabilization, fiber-preserving diffeomorphism, and addition of constants, we can assume that there exist generating families for $L_{1}$ and $L_{2}$ that agree with the same quadratic function outside $K_{1}$ and $K_{2}$. Thus there exists a generating family $F\left(x_{1}, x_{2}, \mathbf{e}\right)$ for $L_{1} \# L_{2}$ so that, for some $\epsilon>0, F\left(x_{1}, x_{2}, \mathbf{e}\right)$ generates the $x_{1}$-translate of $L_{1}$ on $\left\{x_{1}<-\epsilon\right\} ; F\left(x_{1}, x_{2}, \mathbf{e}\right)$ generates the $x_{1}$-translate of $L_{2}$ on $\left\{x_{1}>\epsilon\right\}$, and on $\left|x_{1}\right|<\epsilon, F\left(x_{1}, x_{2}, \mathbf{e}\right)$ agrees with a quadratic function $Q(\mathbf{e})$. It follows that on $x_{1}<-\epsilon, x_{1}$-translates of the gradient trajectories used to define $c_{ \pm}^{L_{1}, a}\left(u_{1}\right)$ will define $c_{ \pm}^{L_{1} \# L_{2}, a}\left(u_{1}, 0\right)$, and hence the value $c_{ \pm}^{L_{1}, a}\left(u_{1}\right)$ agrees with $c_{ \pm}^{L_{1} \# L_{2}, a}\left(u_{1}, 0\right)$. An analogous argument holds on $x_{1}>\epsilon$. The claimed calculation of $c_{ \pm}^{L_{1} \# L_{2}, a}\left(u_{1}, u_{2}\right)$ follows from the definition.

Using this, we can show that the result of repositioning the two figure-8 curves of opposite crossings in Figure 1(d) so that they are configured as in Figure 4 cannot be realized as the diagram of a negative slice of a connect sum of Lagrangians:

Corollary 4.6. For any $A, B>0,8^{-}(A)+8^{+}(B)$ cannot be realized as the diagram of a generic negative slice of any connect sum of flat-at-infinity planar Lagrangians.

Proof. Suppose there exists $L_{1} \# L_{2}$ and an $a<0$ so that $\left(L_{1} \# L_{2}\right)_{a}$ has diagram $8^{-}(A)+8^{+}(B)$. Then the inclusion of the slice induces $j_{a}^{*} u=$ $(u, u)$, for all $u \in H^{0}(L)$. By Lemma 4.5 and Theorem 4.1, it follows that $c_{ \pm}^{L_{1} \# L_{2}, a}\left(j_{a}^{*} u\right)=0$. By index calculations and Lemma $4.2, C_{ \pm}^{L_{1} \# L_{2}, a}\left(j_{a}^{*} u\right)=$ 0 . Thus we get a contradiction to Non-Vanishing.

On the other hand, capacity arguments do not rule out the possibility of $8^{-}(A)+8^{+}(B)$ appearing as the diagram of a negative slice of any flat-atinfinity planar Lagrangian. 
4.4. Obstructions to the Existence of Cobordisms. We continue our exploration of the use of capacities as obstructions by proving parts (1) and (2) of Theorem 1.4. Combining Theorem 4.1 and Monotonicity yields part (1) of the theorem: for all $0<A<B$, we have:

$$
\left[\widetilde{8}^{+}(A)\right] \unlhd\left[\widetilde{8}^{+}(B)\right], \text { while }\left[\widetilde{8}^{+}(B)\right] \unlhd\left[\widetilde{8}^{+}(A)\right],
$$

where $\widetilde{8}^{+}(A), \widetilde{8}^{+}(B)$ are any slices with diagrams $8^{+}(A), 8^{+}(B)$.

To prove part (2), namely that for all positive areas $A$ and $B$,

$$
\left[\widetilde{8}^{+}(A)\right] \not\left[\left[\widetilde{C}^{+-+}(A, B, B)\right] \text { and }\left[\widetilde{C}^{+-+}(A, B, B)\right] \not \Perp\left[\widetilde{8}^{+}(A)\right],\right.
$$

for any lifts $\widetilde{8}^{+}(A)$ and $\widetilde{C}^{+-+}(A, B, B)$, we use Monotonicity, Theorem 4.1, and the following capacity computation:

Lemma 4.7. If $L$ is any flat-at-infinity planar Lagrangian with negative slice $L_{a}$ that has diagram $C^{+-+}(A, B, B)$ then, for $0 \neq u \in H^{0}\left(L_{a}\right), c_{-}^{L, a}(u)=$ $-A$.

Proof. As in the analysis of $C^{-+-}(A, B, B)$ in the proof of Proposition 4.4, the difference function $\Delta_{a}$ has six critical points whose indices and critical values are the same as before, but with the roles of $\mathcal{P}_{+}$and $\mathcal{P}_{-}$reversed. Let $u \in H^{0}\left(L_{a}\right)$. By Lemma 4.2, we know that $c_{+}^{L, a}(u)=0=C_{-}^{L, a}(u)$. By a similar argument to the one in Proposition $4.4, C_{+}^{L, a}(u)=0$. By Non-Vanishing, it must be the case that $c_{-}^{L, a}(u) \neq 0$. Further, since the critical value for both of the index $N$ critical points is $-A$, we must have $c_{-}^{L, a}(u)=-A$, as desired.

The proof of Theorem 1.6(1), namely that the relation $\unlhd$ is not compatible with the addition + , also relies on a capacity computation:

Lemma 4.8. Let $L$ be any flat-at-infinity planar Lagrangian having a generic height $a<0$ so that $L_{a}$ has diagram $8^{+}(A)+8^{+}(A)$. Then, for any nonzero $u \in H^{0}\left(L_{a}\right), c_{-}^{L, a}(u)=-A$.

Proof. For the difference function $\Delta_{a}$, there are four non-degenerate critical points: two in $\mathcal{P}_{+}$with value $A$ and index $N+3$ and two in $\mathcal{P}_{-}$with value $-A$ and index $N$. By these calculations, it follows that for all $u \in H^{0}\left(L_{a}\right)$, $c_{+}^{L, a}(u)=0, C_{+}^{L, a}(u)=0$, and $C_{-}^{L, a}(u)=0$. Then by the Non-Vanishing property of capacities, for any nonzero $u \in H^{0}\left(L_{a}\right), c_{-}^{L, a}(u) \neq 0$. Since $c_{-}^{L, a}(u)$ must be a critical value associated to a critical point of index $N$, we then know that $c_{-}^{L, a}(u)=-A$.

Theorem 4.1, Lemma 4.8, and Monotonicity then imply Theorem 1.6(1): for any $A>0$,

$$
\left[\widetilde{8}^{+}(A)\right] \unlhd\left[\widetilde{8}^{+}(A)\right]+\left[\widetilde{8}^{+}(A)\right] .
$$




\section{5. $\left(\mathcal{S}_{c}, \unlhd\right)$ AS a Partially Ordered Set}

As stated in Theorem 1.4(3), the restriction of the relation $\unlhd$ to the set $\mathcal{S}_{c}$ of connected slices is a partial order, i.e. $\unlhd$ is reflexive, transitive, and antisymmetric. Reflexivity is obvious from the definition. The proofs of transitivity and antisymmetry both require us to glue together two Lagrangians along a common slice. The first step in this endeavor is to prove that any two collar neighborhoods of a generic slice are equivalent:

Lemma 5.1. Let $L, L^{\prime} \subset \mathbb{R}^{4}$ be two flat-at-infinity planar Lagrangians that are transverse to and agree on $\left\{y_{2}=a\right\}$ :

$$
S=L_{a}=L_{a}^{\prime} \text {. }
$$

Then, for all $\epsilon>0$, there exist neighborhoods $V \subset U$ of $S$ in $L$ and a symplectic isotopy $\Phi_{t}$ of $\mathbb{R}^{4}$ so that $\left.\Phi_{t}\right|_{L}$ is the identity on $S$ and on the complement of $U, \Phi_{1}(U) \subset\left\{a-\epsilon<y_{2}<a+\epsilon\right\}$, and $\Phi_{1}(V) \subset L^{\prime}$.

Proof. By a result of Eliashberg and Polterovich [6], there is a symplectomorphism $\psi$ of $\mathbb{R}^{4}$ taking $L$ to $L_{0}$, the zero-section of $\mathbb{R}^{4}=T^{*} \mathbb{R}^{2}$. Let $\gamma$ be the image of $S$ under $\psi$. Let $A$ be the disjoint union of annuli around the components of $S$ in $L^{\prime}$. By the transversality assumption, for sufficiently small $A$, the Lagrangian $G=\psi(A)$ will be the graph of a 1-form, necessarily closed, over a neighborhood $C$ of $\gamma$. This 1-form vanishes on each component of $\gamma$, and so we can assume $G$ is the graph of an exact 1-form $d g$. Since there exists $g$ so that both $g$ and $d g$ vanish on $\gamma$, for an arbitrary $\delta>0$, we can construct a bump function $\rho$ so that $\|d(\rho g)\|<\delta$. Now consider the symplectic isotopy $\varphi_{t}$ of $T^{*} \mathbb{R}^{2}$ given by $\varphi_{t}(\mathbf{x}, \mathbf{y})=(\mathbf{x}, \mathbf{y}+t d(\rho g)(\mathbf{x}))$. We may choose this isotopy to displace $L_{0}$ by as little as we want. Further, we have $\varphi_{t}(\gamma)=\gamma$, $\varphi_{1}\left(L_{0}\right)$ agrees with $G$ in a neighborhood of $\gamma$, and $\left.\varphi_{t}\right|_{L_{0}}=$ id outside $C$. Then $\Phi_{t}=\psi^{-1} \varphi_{t} \psi$ is the desired isotopy since we may assume that we have chosen $C$ and $\delta$ small enough so that $\Phi_{t}(U) \subset\left\{a-\epsilon<y_{2}<a+\epsilon\right\}$.

Thus, we obtain the following gluing construction for flat-at-infinity planar Lagrangians meeting at a connected slice: ${ }^{3}$

Proposition 5.2. Let $L, L^{\prime} \subset \mathbb{R}^{4}$ be two flat-at-infinity planar Lagrangians that are transverse to and agree on $\left\{y_{2}=a\right\}$ :

$$
S=L_{a}=L_{a}^{\prime} \text {. }
$$

If $S$ is connected then, for all $\epsilon>0$, there exists a flat-at-infinity planar Lagrangian $L^{\prime \prime}$ such that:

(1) $L^{\prime \prime} \cap\left\{y_{2}<a-\epsilon\right\}=L \cap\left\{y_{2}<a-\epsilon\right\}$ and

(2) $L^{\prime \prime} \cap\left\{y_{2}>a+\epsilon\right\}=L^{\prime} \cap\left\{y_{2}>a+\epsilon\right\}$.

Proof. The previous lemma shows that we may glue two flat-at-infinity planar Lagrangians $L$ and $L^{\prime}$ along a common slice $S$ to obtain a new flat-atinfinity Lagrangian $L^{\prime \prime}$. The fact that $S$ is connected allows us to use the

\footnotetext{
${ }^{3}$ This is the only place where we use the connectivity assumption.
} 
Jordan curve theorem to show that, topologically, the gluing removes and then replaces a disk from the upper Lagrangian, thus resulting in another planar Lagrangian.

We are now ready to prove transitivity and antisymmetry.

Proof of Transitivity. The only nontrivial case to prove is when $\mathbf{S}_{1} \triangleleft \mathbf{S}_{2}$ and $\mathbf{S}_{2} \triangleleft \mathbf{S}_{3}$. This means that (up to equivalence) there exist flat-at-infinity planar Lagrangians $L$ and $L^{\prime}$ and real numbers $a<b<0$ and $a^{\prime}<b^{\prime}<0$ such that for some representatives $S_{i} \in \mathbf{S}_{i}$,

$$
\begin{array}{ll}
i_{b}\left(S_{2}\right)=L_{b} & i_{b^{\prime}}\left(S_{3}\right)=L_{b^{\prime}}^{\prime} \\
i_{a}\left(S_{1}\right)=L_{a} & i_{a^{\prime}}\left(S_{2}\right)=L_{a^{\prime}}^{\prime} .
\end{array}
$$

By Lemma 2.2, we may assume that $b=a^{\prime}$. We now apply Proposition 5.2 to glue $L$ to $L^{\prime}$ along $S_{2}$ to obtain a flat-at-infinity planar Lagrangian joining $S_{1}$ to $S_{3}$.

Proof of antisymmetry. To prove antisymmetry, we use the capacities defined in Subsection 4.1 and argue by contradiction. Suppose that $\mathbf{S} \unlhd \mathbf{S}^{\prime}$ and $\mathbf{S}^{\prime} \unlhd \mathbf{S}$, but that $\mathbf{S} \neq \mathbf{S}^{\prime}$. It follows that, possibly after an equivalence, there are Lagrangians joining the representative slices $S$ and $S^{\prime}$ (and vice versa). Suppose that the $x_{1} y_{1}$-projection of $S$ has $n$ double points. Using Lemma 2.5 and Proposition 5.2, we may construct a flat-at-infinity planar Lagrangian $L$ with slices $L_{-1} \sim L_{-2} \sim \cdots \sim L_{-(n+1)} \sim S$. Note that this construction yields difference functions $\Delta_{-k}, k=1, \ldots, n+1$, that all have the same $2 j$ nonzero critical values, for some $j \leq n$, with $j$ of them positive and $j$ of them negative. If $u$ is any nonzero class in $H^{*}(L)$ then we claim that $c^{L,-(n+1)}\left(i^{*} u\right)=0$ for all four capacities, a contradiction to the NonVanishing property. To prove the claim, notice that Monotonicity implies that:

$$
c_{-}^{L,-1}\left(i^{*} u\right) \leq c_{-}^{L,-2}\left(i^{*} u\right) \leq \cdots \leq c_{-}^{L,-(n+1)}\left(i^{*} u\right) \leq 0 .
$$

Since these capacities can only take on the $j \leq n$ negative critical values or 0 and each negative critical value can occur at most once, we must have $c_{-}^{L,-(n+1)}\left(i^{*} u\right)=0$. An analogous argument applied to

$$
C_{+}^{L,-1}\left(i^{*} u\right) \geq C_{+}^{L,-2}\left(i^{*} u\right) \geq \cdots \geq C_{+}^{L,-(n+1)}\left(i^{*} u\right) \geq 0
$$

shows that $C_{+}^{L,-(n+1)}\left(i^{*} u\right)=0$, and Lemma 4.3 shows that the other two capacities vanish.

\section{REFERENCES}

1. V. I. Arnold, Lagrange and Legendre cobordisms. I, Funktsional. Anal. i Prilozhen. 14 (1980), no. 3, 1-13, 96.

2. M. Audin, Cobordismes d'immersions lagrangiennes et legendriennes, Travaux en Cours [Works in Progress], vol. 20, Hermann, Paris, 1987. 
3. Yu. Chekanov, Lagrangian embeddings and Lagrangian cobordism, Topics in singularity theory, Amer. Math. Soc. Transl. Ser. 2, vol. 180, Amer. Math. Soc., Providence, RI, 1997, pp. 13-23.

4. Ya. Eliashberg, Cobordisme des solutions de relations différentielles, South Rhone seminar on geometry, I (Lyon, 1983), Travaux en Cours, Hermann, Paris, 1984, pp. 17-31.

5. __ Topology of 2-knots in $\mathbf{R}^{4}$ and symplectic geometry, The Floer memorial volume, Progr. Math., vol. 133, Birkhäuser, Basel, 1995, pp. 335-353.

6. Ya. Eliashberg and L. Polterovich, Local Lagrangian 2-knots are trivial, Ann. of Math. (2) 144 (1996), no. 1, 61-76.

7. J. Sabloff and L. Traynor, Capacities for slices of unknotted planar Lagrangians, Preprint, 2008.

8. C. Viterbo, Symplectic topology as the geometry of generating functions, Math. Ann. 292 (1992), no. 4, 685-710.

Haverford College, Haverford, PA 19041

E-mail address: peiseman@haverford.edu

Haverford College, Haverford, PA 19041

E-mail address: jlima@haverford.edu

Haverford College, Haverford, PA 19041

E-mail address: jsabloff@haverford.edu

Bryn Mawr College, Bryn Mawr, PA 19010

E-mail address: 1traynor@brynmawr.edu 